\title{
Efficacy and Safety of Anti-D for Acute Immune Thrombocytopenic Purpura in Children in El-Nasr Hospital - Case Report
}

\author{
Amal Shorbasy $^{1}$, Heba Makki ${ }^{2 *}$ and Mustafa El Kahlout ${ }^{3}$ \\ ${ }^{1}$ Pharmacist, Masterin Pharmaceutical Sciences, El-Nasr Pediatric Hospital, \\ Gaza,Palestine \\ ${ }^{2}$ Pediatrician, Palestinian Board of Pediatrics, El-Nasr PediatricHospital, \\ Gaza,Palestine \\ ${ }^{3}$ Consultant Pediatrician, Palestinian Board of Pediatrics,Director of El Nasr \\ Pediatric Hospital, Gaza, Palestine
}

*Corresponding Author: Heba Makki, Pediatrician, Palestinian Board of Pediatrics, El-Nasr PediatricHospital, Gaza, Palestine.

DOI: $10.31080 /$ ASPE.2020.03.0269

\begin{abstract}
Immune (idiopathic) thrombocytopenic purpura (ITP) of childhood is characterized by acquired thrombocytopenia. ITP seems to occur more often after a viral respiratory illness. Findings on physical examination are normal, other than the finding of petechiae and purpura.

Anti-D is effective in the treatment of children with ITP (Rh-positive non-splenectomized patients) and with higher doses appears to have comparable results to IVIG. Our experience in treating children with ITP in Gaza strip hospitals is restricted to use of corticosteroids or IVIG. No published clinical trial on safety and efficacy of anti-D in acute ITP in children in the Gaza strip.

Four cases ( 2 males and 2 females) were admitted to El-Nasr Pediatric hospital during the period from April 2017 to December 2017 and all of them were diagnosed as acute ITP. The youngest patient was 1 year and 10 months old and the oldest patient was 11 years. Clinical presentation included skin rash 3 caseswith oral cavity bleeding in one case. All cases had history of previous URTI 1-2 weeks before the attack of ITP.Anti-D was used for allthese cases. Dose given was $50-75 \mathrm{mic} / \mathrm{kg}$. The overall response rate was $75 \%$, three cases showed good response and platelet count was elevated within 24 hours. The duration of hospitalization for all the responsive cases was 2 days. No side effects of Anti- D were noted.

Conclusion: Anti-D is used in pediatric age group for use in some carefully selected cases of acute ITP. Anti-D showed nearly the same efficacy and safety with cost effectiveness comparable to IVIG.
\end{abstract}

Keywords: Idiopathic Thrombocytopenic Purpura; Anti-D; IVIG

\section{Introduction}

Immune(idiopathic) thrombocytopenic purpura (ITP) of childhood is characterized by acquired thrombocytopenia and is a generally benign disorder of unknown cause [3]. In about 60 percent of children with ITP, there is a history of a prior infection.

The peak age is 1-4 years, although the age ranges from early in infancy to the elderly. In childhood, males and females are equally affected. ITP seems to occur more often in late winter and spring after the peak season of viral respiratory illness.

Findings on physical examination are normal, other than the finding of petechiae and purpura. Splenomegaly, lymphadenopathy, bone pain and pallor are rare[1].

In 2010, an international consensus report recommended that the choice of treatment be based upon factors that includes clinical 
symptoms, circulating platelet count, and the impact of ITP upon the patient's quality of life. Treatment is mainly supportive. When it is decided to use pharmacologic therapy, treatment options include corticosteroids, intravenous immunoglobulin (IVIG) or intravenous anti-Rho(D) immune globulin. Several studies have shown that the duration of symptomatic thrombocytopenia is shortened by any of these three modalities compared to no treatment[3].

Anti-D is a fraction of the gamma globulin antibodies that are derived from blood plasma and is effective in the treatment of children with ITP [4]. Withhigher doses, Anti-D appears to have comparable results to IVIG.

Our experience in treating children with ITPin Gaza strip hospitals is limited to the use corticosteroids or IVIG. Corticosteroids have many side effects on children and IVIG is very expensive and not always available. For these reasons, Anti -D was decided to be used for some selected patients. This study was planned to show safety and efficacy of anti-D in treatment ofacute ITP in children of the Gaza Strip as there are no published clinical trials on this issue in our region.

\section{Case Report}

The case report was done in El Nasr pediatric hospital in the Gaza Strip-Palestine in the period from April 2017 to August 2017. It was constructed to evaluate the effectiveness and safety of the use of Anti-D for Acute Immune Thrombocytopenic Purpura.

Inclusion criteria wereITP children with Platelet counts $<20,000$ cell $/ \mathrm{mm}^{3}$, Rh-positive blood group and non-splenectomized children with Hemoglobin levelsnot below 10g/dl.

Children were admitted to the pediatric departments after history takingwas done to ensure there is no history of hemolysis. Laboratory testing (Baseline Complete blood count (CBC) and urine analysis, blood group and $\mathrm{Rh}$ ) were done and thengiven the treatment with Anti-D (50-75 micro/kg) intravenously single dose over 5 minutes.

After receiving the Anti-D, the children were clinically followed up by physicians concerning the vital signs, hemodynamic state, fever, allergy or other side effects.
Follow up CBC and urine analysis within 24 hours of Anti-D administration were done and the physical examination done daily for each patient.

Response (R)was defined as gradual elevation of platelet count at least two fold increase from the baseline count within 12 hours. Non-response (NR) was defined as no elevation or decrease platelet count within 12 hour.

Follow up of our patients clinically and laboratory (CBC) after 1 week of discharge were done.

\section{Results}

Four cases (2 males and 2 females) were admitted to El-Nasr pediatric hospital during ourstudy period, all of them were diagnosed as acute ITP and received Anti-D. The youngest patient was 1 year and 10 months old and the oldest patient was 11 years. Clinical presentation included skin rash inthe 3 caseswith skin rash and oral cavity bleeding in one case. All cases had history of previous upper respiratory tract infection (URTI) 1-2 weeks before the attack of ITP.

Anti-D was used for all the four cases. Dose given was 50-75mic/ $\mathrm{kg}$. The overall response rate was $75 \%$, as three cases showed response (R) and platelet count was elevated within 12 hours.

The three successful cases showed elevation of platelet count during the hospitalization period and 1 week after discharge (Figure 1) and no significant hemolysis or drop of hemoglobin (Figure 2).

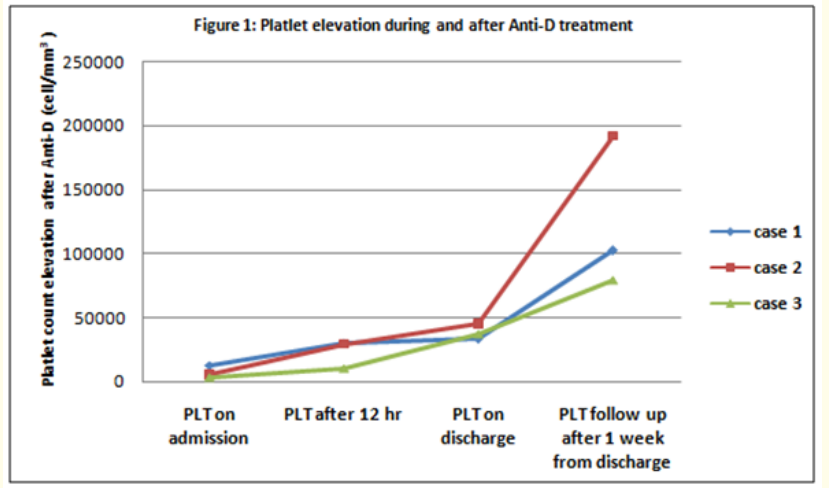

Figure 1: Platelet evaluation during and after anti-D treatment. 


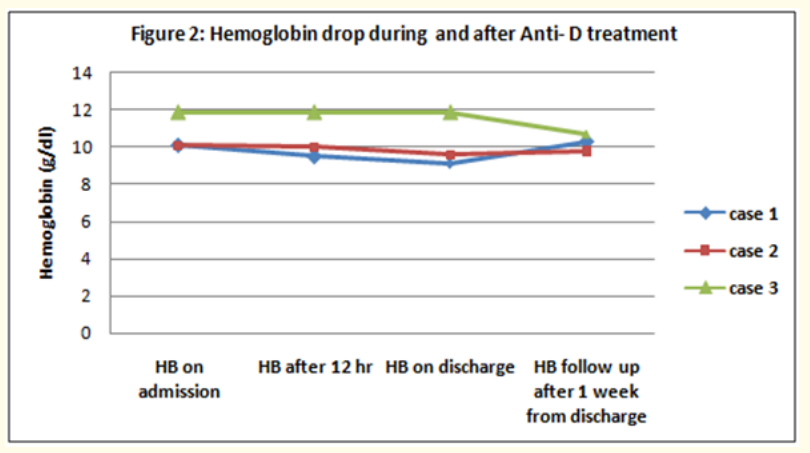

Figure 2: Hemoglobin drop during and after anti-D treatment.

\section{Discussion}

IV Anti- D showed an efficacy and safety in treatment for acute ITP in children [10].

The presumed mechanism of action of anti-D immunoglobulin in ITP involves extravascular hemolysis of anti-D-sensitized red blood cells (RBCs) by splenic macrophages, which results in decreased splenic sequestration of autoantibody-sensitized platelets and an increased platelet count [2] and this explains why Anti-D is used and effective in only Rh- positive non splenectomized patients.

In the Gaza strip, ITP cases may present with sometimes dangerous very low levels of platelets. Some of these cases are at risk of bleeding if not managed with steroids or IVIG. IVIG is not always available and has high cost especially if our patient's weight is high, necessitating large doses of IVIG.

Also, we need IVIG for treatment of other diseases like GuillainBarré Syndrome and Kawasaki disease in which IVIG is curative and prevents serious complications.Anti -D is very effective in raising platelet count as its efficacyreaches $80 \%$ in raising platelets count in first 3-4 days. This is nearly similar to our case results as 3 cases out of 4 cases (75\%) showed platelet count elevation. IVantiD treatment compares favorably with IVIGwhere Anti-D has lower cost than IVIG [2]. IVIG dose in ITP is 1 gram per kg and may be needed to be repeated in the next day if no response noted. On the other hand, Anti -D is given only once. Thethree cases (Weights: $11 \mathrm{~kg}, 9 \mathrm{~kg}, 33 \mathrm{~kg}$ ) needed 10 syringes of Anti-D.The cost of IVIG dose in this situation is higher 10 times than the cost of Anti-D dose. Also,IVIGneeds slow infusion rate, initial infusion rate is $0.5 \mathrm{mg} /$ $\mathrm{kg} / \mathrm{min}$. Maximum infusion rate is $4 \mathrm{mg} / \mathrm{kg} / \mathrm{min}$ (i.e. $4-6$ hours or longer according to the weight of the child) to prevent infusion reactions and complications. In contrast, Anti- D is given in one dose over 3-5 minutes only. The most frequent Anti -D related adverse events are chills, pyrexia, drop in hemoglobin and increase in bilirubin [11]. Both IVIG and Anti- D has side effects and precautions like all other medications. Both needs follow up of patients post injection.

However, major side effects reported for Anti -D includes prolonged intravascular hemolysis $[3,9,10]$. Thus, care should be taken while using the drug in patients with low hemoglobin[2]. Our study showed no significant drop in hemoglobin noted as shown in figure

2.

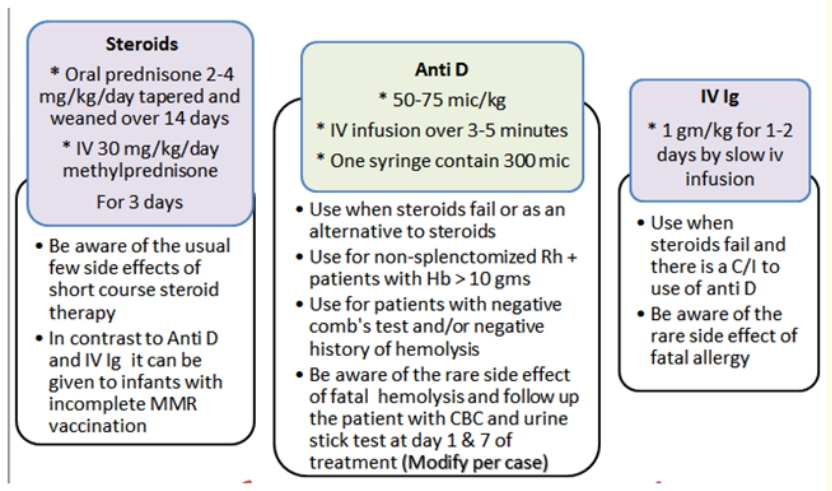

Figure 3: El-Nasr pediatric hospital protocol of management of cases of ITP in children [8].

\section{Conclusion}

Anti D is used in pediatric age group for use in some carefully selected cases of acute ITP. Anti D showed nearly the same efficacy and safety with cost effectiveness comparable to IVIG.

\section{Bibliography}

1. Robert M Kliegman MD. Nelson textbook $20^{\text {th }}$ edition (2016).

2. Rahul Naithani., et al. "Efficacy and Safety of Anti-D for Immune Thrombocytopenic Purpura in Children". All India Institute of Medical Sciences, New Delhi, India. Indian Pediatrics 47 (2010).

3. "Rho (D) immune globulin: Drug information". Up To Date version 21.6. 
4. James N George., et al. "Intravenous Anti-D: another treatment for ITP, ITP support association” (2003).

5. Eric Cheung and Howard A Liebman. "Anti-RhD immunoglobulin in the treatment of immune thrombocytopenia". Biologics Journal 3 (2019): 57-62.

6. Aziz Eghbali., et al. "Comparison between IV immune globulin (IVIG) and anti-D globulin for treatment of immune thrombocytopenia: a randomized open-label study". Fundamental and Clinical Pharmacology 30 (2016): 385-389.

7. Joanne Yacobovich., et al. "Anti-D treatment for pediatric immune thrombocytopenia: Is the bad reputation justified?" Seminares in Hematology Journal 53 (2016): S64-S66.

8. Awadallah M. "Pediatric landmarks" (2019).

9. HyperRHO S/D Full Dose (Rho[D] Immune Globulin. Research Triangle Park, NC: Grifols Therapeutics (2012).

10. Neunert C., et al. "The American Society of Hematology 2011 evidence-based practice guideline for immune thrombocytopenia”. American Society of Hematology 117.16 (2011): 41904207.

11. Rhophylac (Rho[D] Immune Globulin). Kankakee, IL: CSL Behring (2016).

\section{Assets from publication with us}

- Prompt Acknowledgement after receiving the article

- Thorough Double blinded peer review

- Rapid Publication

- Issue of Publication Certificate

- High visibility of your Published work

Website: www.actascientific.com/

Submit Article: www.actascientific.com/submission.php

Email us: editor@actascientific.com

Contact us: +919182824667 